\title{
THE CRYSTALLIZATION OF A TIN-BEARING GRANITOID SUITE: THE BUSHVELD GRANITES IN THE ZAAIPLAATS AREA SOUTH AFRICA
}

\author{
JUSSI TUOMAS OLLILA
}

OLLILA, JUSSI TUOMAS, 1984: The crystallization of a tin-bearing granitoid suite: The Bushveld granites in the Zaaiplaata are, South Africa. Bull. Geol. Soc. Finland 56, Part 1-2, 75-88.

Alkali feldspars from the tin-bearing Bobbejaankop and Lease granites, and from the surrounding barren Main granite in the Zaaiplaats tin-field were studied by X-ray diffraction method. The temperatures and oxygen fugacities of the lowermost Main granite were calculated using quantitative microprobe analyses from the coexisting iron-titanium minerals.

The alkali feldspars in the Bobbejaankop granite and in the upper layers of the Main granite are invariably high ordered maximum microclines whereas those in the coarse-grained bottom layers of the Main granite have a high thermal state, i.e. they are orthoclases. The alkali feldspars in the Lease granite are intermediate microclines.

The subsolidus reequilibration of iron-titanium oxides ceased at a very low temperature $\left(382.8^{\circ} \mathrm{C}\right)$ in the lower Main granite which crystallized under variable oxygen fugacity in the temperature range $500^{\circ}$ to $650^{\circ} \mathrm{C}$, and at least partially, under reducing conditions. Oxidizing conditions prevailed at temperatures higher than $650^{\circ} \mathrm{C}$.

The alkali feldspars in the lower Main granite are obviously original magmatic phases and the absence of a volatile phase at the time of initial crystal growth may have prevented the later development of triclinic alkali feldspars. The feldspars in the upper layers of the Main granite have apparently undergone thorough subsolidus exsolution equilibrium recrystallization due to the progressively more hydrous interstitial melt that contributed towards the high degree of ordering.

Maximum microclines in the Bobbejaankop granite are due to the presence of aqueous, high-saline fluid phase during its crystallization. The occurrence of intermediate microclines in the Lease granite indicates quenching and a faster cooling rate than that of the surrounding rocks.

Key words: Bushveld granites, structural state of alkali feldspar, geothermometry, oxygen barometry, iron-titanium oxides, fractional crystallization, intercumulus melt, hydrothermal granitization.

Jussi Tuomas Ollila: Geological Survey of Finland, P.O. Box 77, 96101 Rovaniemi 10, Finland.

\section{Introduction}

The acid phase of the Bushveld Complex in the Zaaiplaats tin-mining area, $30 \mathrm{~km}$ northwest of Potgietersrus in the Central Transvaal, South
Africa, displays a variety of intrusive granitic rocks, viz: (i) a stratiform suite of granitoids, called the Main Granite, (ii) the Foothills Granite, and (iii) the Bobbejaankop Granite.

The Foothills granite intrudes into the Main 
granite, the Transvaal sediments, and the Bushveld Complex norite as thin sills and dykes. The Main granite which has intruded along the contact of the Transvaal sediments and overlying felsite as a composite sheet 2660 metres thick has a pseudostratified appearance due to the following textural varieties, from top to base: (i) contact microgranite, (ii) granophyric granite succeeded by, or intercalated with, granophyre and granite-porphyry, (iii) coarse-grained grey, leucocratic granite, and (iv) coarse grained, grey, mesotype granite (Strauss 1954).

The youngest of the granites, the stanniferous Bobbejaankop granite, is always associated with its fine-grained metasomatised roof facies, the Lease Granite, the upper part of which contains the almost always persistent zone of flat, concordant contact pegmatite bodies. The red, coarse-grained Bobbejaankop granite intruded into the Main granite as dome-like plutons. In the Zaaiplaats area it crops out as an elongate body bounded in the southwest by the Lease microgranite and elsewhere by the coarse-grained facies of the stratiform Main granite at the lowermost levels and by the granophyric variety at the higher levels.

\section{Structural state and composition of alkali feldspars}

The objective of the structural state and composition study of alkali feldspars was to test the validity of the method and its feasibility for find-

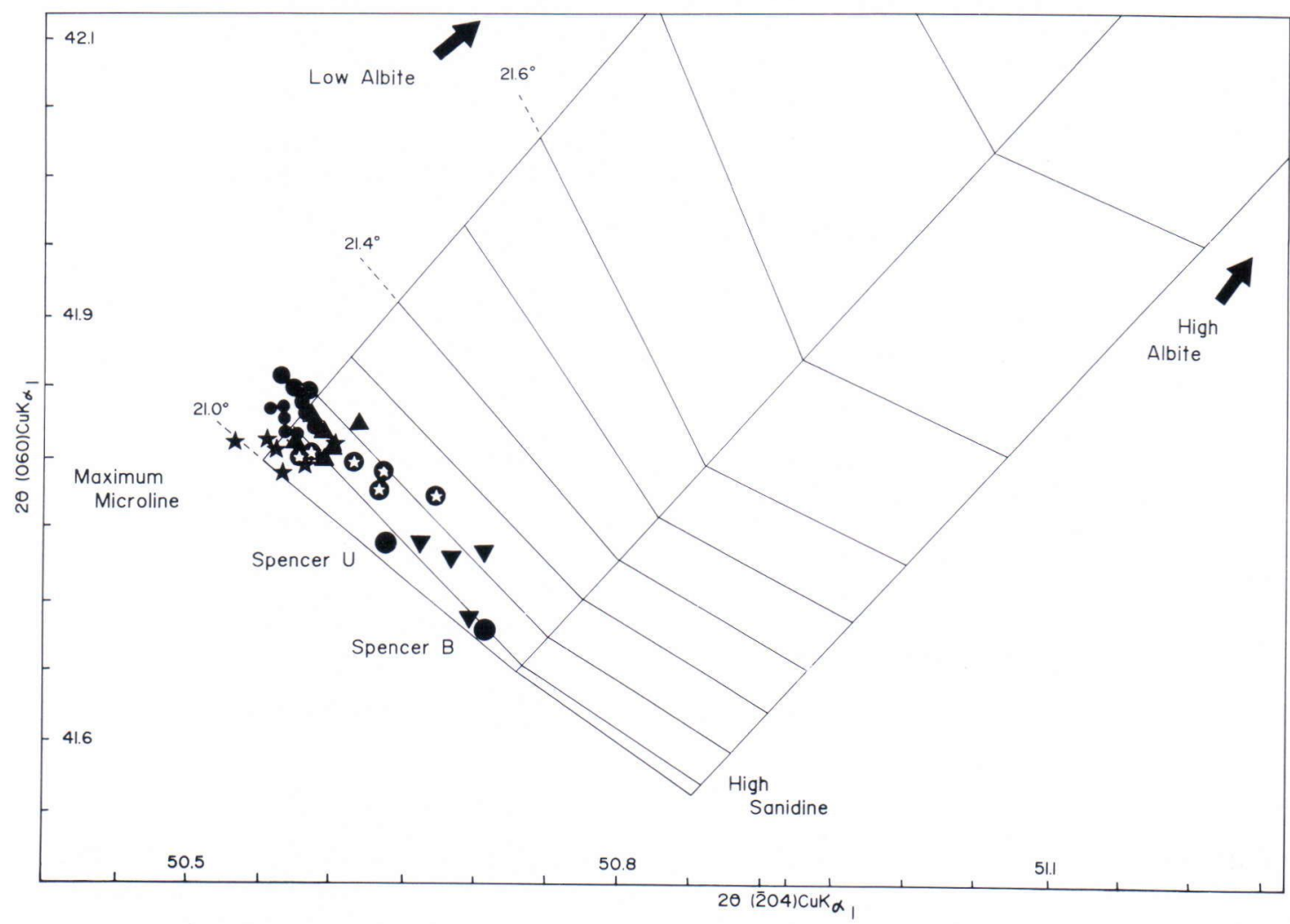

Fig. 1. Observed $2 \Theta$ values of $(060)$ plotted against the $2 \theta$ values of (204) for alkali feldspars in the diagram of Wright (1968). = Bobbejaankop granite (Profile), $\boldsymbol{*}=$ Bobbejaankop granite (Mineralized), $\boldsymbol{Q}=$ Lease granite, $\boldsymbol{\Delta}=$ Main granite (triclinic), and $\boldsymbol{\nabla}=$ Main granite (monoclinic). 
ing differences in alkali feldspars in different granites, and in particular, between the mineralized and barren Bobbejaankop granite.

The profile of samples, marked from $78 / 15 / 1$ up to $78 / 15 / 10$, represent a sequence of samples in a vertical section from the bottom, the barren Bobbejaankop granite through the disseminated cassiterite zone up to the Lease granite contact. The interval between the specimens in the vertical section is about 32 metres. Sample 78/15/10 is from the disseminated cassiterite zone. Six samples, viz. 06377-288, $-300,-24,-108,-154$ and -20 are alkali feldspars from the mineralized pods and patches in the Bobbejaankop granite, where they were always in intimate association with cassiterite. In the Lease granite the alkali feldspars were collected from unmineralized lo- calities. The specimens from the Main granite were selected to represent all the main variations in the layers with different textures and grain sizes in the vicinity of the Zaaiplaats mine.

The cell dimensions for 25 alkali feldspars were determined from diffractometer data using Fe radiation $(\lambda=1.9373 \AA$, $50 \mathrm{kv}, 20 \mathrm{~mA})$. Two scans from 15 to 67 degrees $2 \Theta$ were recorded for each specimen at a goniometer speed of $0.5^{\circ}$ per minute.

The X-ray diffraction patterns were indexed and $2 \theta$ values for the indexed peaks were subsequently used in a cell refinement programme of Appleman and Evans (1973). Because many of the X-ray powder patterns were fairly diffuse, provisional cell parameters were first calculated. The indexing was checked by inspection

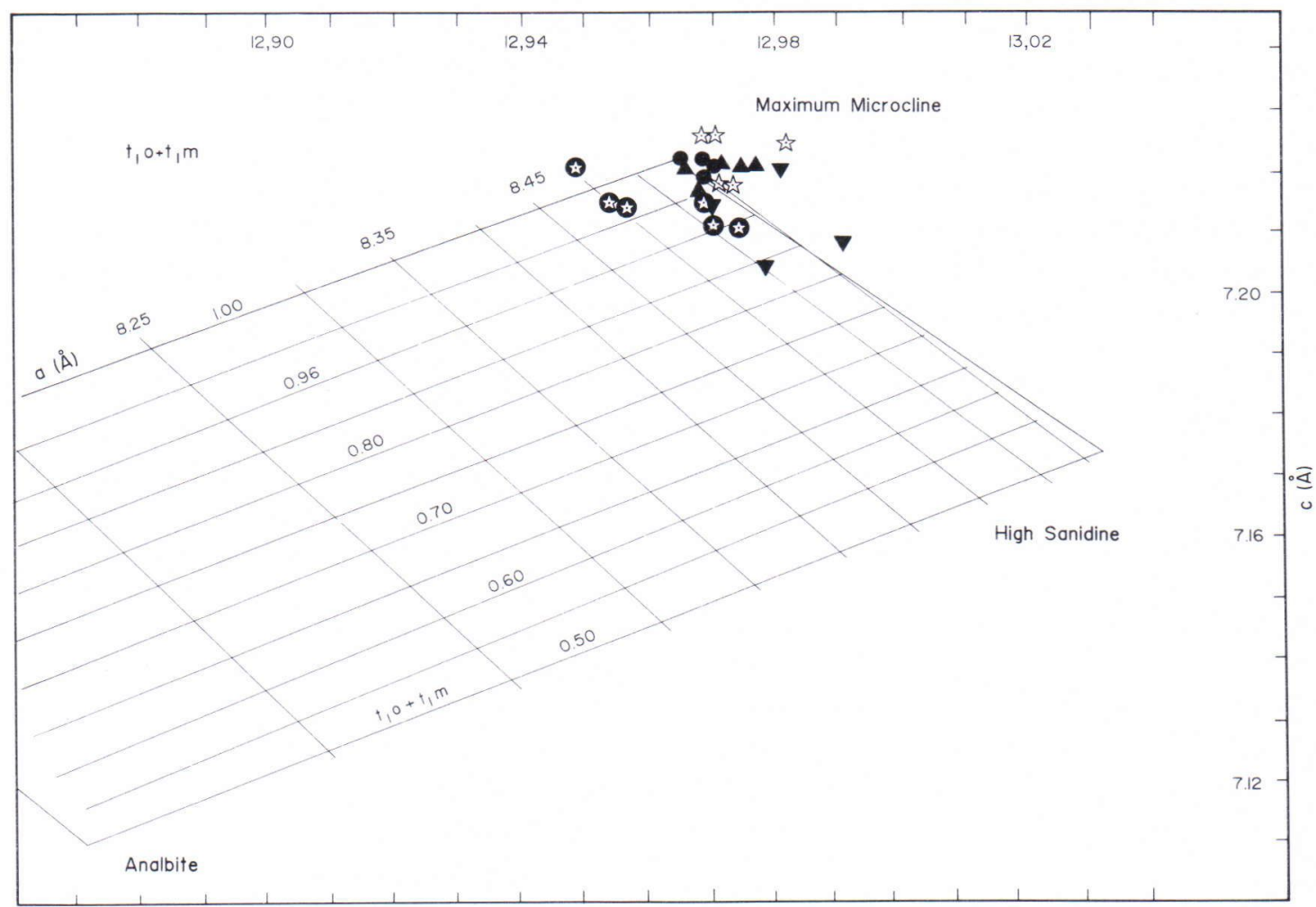

b, (Å)

Fig. 2. The $b-c$ plot of Stewart and Wright (1974) for alkali feldspars. Lines of equal Al-content in $T_{1}$ sites ( $\left.t_{1} \mathrm{O}+\mathrm{t}_{1} \mathrm{~m}\right)$ are based on complete disorder in the analbite - sanidine series and complete order in the low albite - maximum microcline series. Marks as in Fig. 1. 
of the powder patterns, and the indexed peaks were calibrated using quartz as an internal standard, and the final refined unit cell parameters were computed from the inspected powder diffraction data.

The composition and structural state of alkali feldspars that are not strained, can be deter-

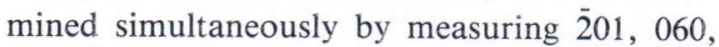
and 204 reflections according to the simple "three-peak» method of Wright (1968). The $2 \theta^{\circ} \mathrm{CuK} \alpha$ values for 060 and 204 are plotted against each other in Fig. 1.

The $\mathrm{b}-\mathrm{c}$ plot (Fig. 2) gives total $\mathrm{Al}$ in the $\mathrm{T}_{1}$ sites $\left(\mathrm{t}_{1} \mathrm{O}+\mathrm{t}_{1} \mathrm{~m}\right)$ and total $\mathrm{Al}$ in the $\mathrm{T}_{2}$ sites, assuming that $t_{2} \mathrm{O}$ is always equal to $t_{2} \mathrm{~m}$, i.e., $t_{2} \mathrm{O}$ $=\mathrm{t}_{2} \mathrm{~m}=1 / 2\left(1.0-\mathrm{t}_{1} \mathrm{O}-\mathrm{t}_{1} \mathrm{~m}\right)$, (Stewart and Ribbe 1969). Even though the $b-c$ plot provides a characterization of structural states, it doesn't distinguish between the one- and two-step ordering trends. Distortion in certain strained alkali feldspars which are strained can be characterized using the contours for $a$ on the $b-c$ plot (Wright and Stewart 1968). Wright and Stewart defined an alkali feldspar as having anomalous cell dimensions when the quantity [a(observed) - a(estimated from $b$ and c)] exceeds $0.02 \AA$. The estimate of $\Delta \mathrm{a}$ is obtained from the contours of Fig. 2, and the values are given in Table 1. However, with the exception of sample 06377-45 from the Lease granite the alkali feldspars studied do not show any significant strain in lattices.

As shown by Stewart and Ribbe (1969), the $\alpha^{*}-\gamma^{*}$ plot (Fig. 3) can be used for a quantitative measure of the difference in $\mathrm{Al}$-contents of the $\mathrm{T}_{1} \mathrm{O}$ and $\mathrm{T}_{1} \mathrm{~m}$ sites. The $\mathrm{t}_{1} \mathrm{o}, \mathrm{t}_{1} \mathrm{~m}$ and $\mathrm{t}_{2} \mathrm{O}=$ $\mathrm{t}_{2} \mathrm{~m}$ values in Table 1 have been calculated according to the relation of Stewart (1975), where:

$$
\begin{aligned}
& 1.0-\left(\mathrm{t}_{1} \mathrm{O}+\mathrm{t}_{1} \mathrm{~m}\right)=\mathrm{t}_{2} \mathrm{O}+\mathrm{t}_{2} \mathrm{~m}, \text { and } \\
& \mathrm{t}_{2} \mathrm{O}=\mathrm{t}_{2} \mathrm{~m}=\left(\mathrm{t}_{2} \mathrm{O}+\mathrm{t}_{2} \mathrm{~m}\right) / 2 .
\end{aligned}
$$

The alkali feldspars in the Bobbejaankop granite exhibit the highest degree of ordering of the specimens studied. According to the 060 204) peak plot and the $b-c$ plot they are maxi- mum microclines indicating thus the lowest thermal state.

The Bobbejaankop granite specimens in association with cassiterite in the mineralized pods and patches of the disseminated zone marked as »mineralized» in Table 1, are not marked different from those below the disseminated zone marked as »profile».

The high $t_{1} m$ values (between 0.44 and 0.49 ) (Table 1) demonstrate clearly a more complicated ordering path than the »one-step ordering process» (Stewart 1975) for these alkali feldspars.

The powder patterns of the Lease granite alkali feldspar specimens were very diffuse, and the crystallographic data in Table 1 cannot be regarded as very informative. However, the "sthree-peak method» of Wright (1968) could be employed to show that at least four of the six specimens studied are intermediate microclines, a trend that is confirmed by the $b-c$ plot and the $\alpha^{*}-\gamma^{*}$ plot (Fig. 3).

The $\mathrm{t}_{1} \mathrm{~m}$ values (between 0.29 and 0.36 ) and the $\Delta 2 \theta \mathrm{CuK} \alpha(\overline{2} 04-060)$ values (between 8,785 and 8,855 ) (Table 1) are reasonably close to reference values of intermediate microcline Spencer $\mathrm{U}, 0.235$ and 8.83 respectively.

The four monoclinic alkali feldspars from the lower portions of the stratiform Main granite are probably structurally heterogeneous, because their ordering parameters $t_{1} \mathrm{O}$ (between 0.42 and 0.47 ), $\mathrm{t}_{2} \mathrm{O}=\mathrm{t}_{2} \mathrm{~m}$ (between 0.04 and 0.08 ), and $\Delta 2 \Theta \mathrm{CuK} \alpha(\overline{2} 04-060)$ (between 8.830 and 8.995) (Table 1) are fairly near the reference values of adularia, viz. $0.405,0.08$, and 8.90 respectively. The ordering parameters $\mathrm{t}_{1} \mathrm{O}+$ $\mathrm{t}_{1} \mathrm{~m}$ (between 0.84 and 0.93 ) and $\mathrm{t}_{1} \mathrm{~m}$ (between 0.21 and 0.23 ) are close to the values of intermediate microcline, Spencer U, viz. 0.86 and 0.235 , respectively.

The crystallographic data on the five triclinic specimens from the upper parts of the Main granite are essentially similar to those of the Bobbejaankop granite, the $t_{1} \mathrm{o}-\mathrm{t}_{1} \mathrm{~m}$ values being slightly higher. 
Table 1. Crystallographic data for alkali feldspars (estimated standard deviations in parentheses).

\begin{tabular}{|c|c|c|c|c|c|c|c|c|c|c|}
\hline Specimen & $\mathrm{a}$ & b & $\mathrm{c}$ & $\alpha$ & $\beta$ & $\gamma$ & $\alpha^{*}$ & $\gamma^{*}$ & \multicolumn{2}{|c|}{ Volume $\AA^{3}$} \\
\hline \multicolumn{11}{|c|}{ Bobbejaankop granite - profile. } \\
\hline $78 / 15 / 1$ & $8.589(3)$ & $12.968(4)$ & $7.219(2)$ & $90.51(5)$ & $115.96(3)$ & $87.98(4)$ & $90.39(4)$ & $91.99(4)$ & \multicolumn{2}{|c|}{$722.52(34)$} \\
\hline $78 / 15 / 4$ & $8.597(5)$ & $12.965(6)$ & $7.223(3)$ & $90.55(5)$ & $116.03(5)$ & $87.93(5)$ & $90.39(5)$ & $92.03(5)$ & \multicolumn{2}{|c|}{$722.93(49)$} \\
\hline $78 / 15 / 7$ & $8.595(4)$ & $12.970(4)$ & $7.222(2)$ & $90.59(5)$ & $116.03(4)$ & $87.94(5)$ & $90.34(5)$ & $91.97(4)$ & \multicolumn{2}{|c|}{$722.93(38)$} \\
\hline $78 / 15 / 10$ & $8.596(7)$ & $12.968(6)$ & $7.223(2)$ & $90.58(7)$ & $116.03(4)$ & $87.89(6)$ & $90.38(5)$ & $92.06(5)$ & \multicolumn{2}{|c|}{$722.97(53)$} \\
\hline \multicolumn{11}{|c|}{ Bobbejaankop granite - mineralized. } \\
\hline $06377-288$ & $8.603(3)$ & $12.971(3)$ & $7.219(1)$ & $90.84(3)$ & $115.95(2)$ & $87.66(3)$ & $90.20(3)$ & $92.19(3)$ & \multicolumn{2}{|c|}{$723.76(24)$} \\
\hline $06377-300$ & $8.582(9)$ & $12.970(5)$ & $7.227(2)$ & $90.56(6)$ & $115.96(4)$ & $88.01(7)$ & $90.34(4)$ & $91.94(5)$ & \multicolumn{2}{|c|}{$722.75(69)$} \\
\hline $06377-24$ & $8.621(7)$ & $12.983(2)$ & $7.225(1)$ & $92.03(8)$ & $116.08(3)$ & $86.37(4)$ & $89.51(3)$ & $93.05(3)$ & \multicolumn{2}{|c|}{$725.11(45)$} \\
\hline $06377-108$ & $8.595(5)$ & $12.970(4)$ & $7.227(2)$ & $90.59(7)$ & $115.97(3)$ & $87.89(5)$ & $90.37(5)$ & $92.0(5)$ & \multicolumn{2}{|c|}{$723.78(42)$} \\
\hline $06377-154$ & $8.586(5)$ & $12.968(3)$ & $7.227(1)$ & $90.62(3)$ & $116.06(6)$ & $87.96(4)$ & $90.30(3)$ & $91.95(3)$ & \multicolumn{2}{|c|}{$722.36(37)$} \\
\hline $06377-20$ & $8.578(6)$ & $12.973(5)$ & $7.218(2)$ & $90.48(5)$ & $115.91(3)$ & $88.10(7)$ & $90.39(4)$ & $91.88(7)$ & \multicolumn{2}{|c|}{$722.06(45)$} \\
\hline Lease gran & & & & & & & & & & \\
\hline $06377-45$ & $8.383(25)$ & $12.970(4)$ & $7.211(2)$ & $90.38(7)$ & $115.82(6)$ & $88.68(10)$ & $90.21(7)$ & $91.28(11)$ & 705. & $.57(20)$ \\
\hline $06377-139$ & $8.539(20)$ & $12.968(14)$ & $7.215(6)$ & $90.26(16)$ & $115.86(15)$ & $89.12(20)$ & $90.14(13)$ & $90.85(17)$ & 718.8 & $82(18)$ \\
\hline $06377-143$ & $8.448(31)$ & $12.957(9)$ & $7.214(5)$ & $90.14(10)$ & $115.91(14)$ & $89.20(13)$ & $90.22(8)$ & $90.81(11)$ & 710.2 & $.24(22)$ \\
\hline $06377-144$ & $8.491(55)$ & $12.954(17)$ & $7.215(9)$ & $90.22(20)$ & $115.87(20)$ & $89.16(25)$ & $90.16(16)$ & $90.81(11)$ & 714. & $.07(40)$ \\
\hline $06377-146$ & $8.604(18)$ & $12.948(12)$ & $7.222(6)$ & $90.24(12)$ & $116.26(13)$ & $89.05(17)$ & $90.12(11)$ & $90.82(22)$ & 721. & $.50(14)$ \\
\hline $06377-160$ & $8.564(29)$ & $12.974(12)$ & $7.210(5)$ & $90.05(15)$ & $116.12(12)$ & $89.31(18)$ & $90.28(15)$ & $90.90(16)$ & 719. & $.20(21)$ \\
\hline Main grani & - triclinic. & & & & & & & & & \\
\hline SA $62 / 1$ & $8.586(2)$ & $12.974(2)$ & $7.221(1)$ & $90.74(2)$ & $115.97(2)$ & $87.69(2)$ & $90.30(2)$ & $92.21(2)$ & 722. & $.52(19)$ \\
\hline SA $62 / 1$ & $8.560(10)$ & $12.969(6)$ & $7.217(3)$ & $90.62(9)$ & $115.87(6)$ & $87.87(7)$ & $90.34(7)$ & $92.07(5)$ & 720. & $.44(77)$ \\
\hline SA $60 / 4$ & $8.592(2)$ & $12.977(3)$ & $7.221(1)$ & $90.83(3)$ & $115.98(2)$ & $87.67(2)$ & $90.21(3)$ & $92.18(2)$ & 723. & $.10(21)$ \\
\hline SA $88 / 5$ & $8.593(4)$ & $12.972(4)$ & $7.222(2)$ & $90.80(5)$ & $116.03(3)$ & $87.67(4)$ & $90.24(4)$ & $92.19(3)$ & 722. & $.74(32)$ \\
\hline SA $36 / 7$ & $8.600(3)$ & $12.966(2)$ & $7.221(1)$ & $90.78(2)$ & $116.04(2)$ & $87.75(2)$ & $90.22(2)$ & $92.12(2)$ & 723. & $.04(24)$ \\
\hline Main grani & monoclini & & & & & & & & & \\
\hline SA $76 / 8$ & $8.605(6)$ & $12.992(2)$ & $7.209(2)$ & 90.00 & $115.93(4)$ & 90.00 & 90.00 & 90.00 & 724. & $.71(51)$ \\
\hline SA $90 / 8$ & $8.577(5)$ & $12.981(4)$ & $7.211(1)$ & 90.00 & $115.90(3)$ & 90.00 & 90.00 & 90.00 & 722. & $26(36)$ \\
\hline $\mathrm{SA} 14 / 10$ & $8.591(5)$ & $12.978(4)$ & $7.204(2)$ & 90.00 & $115.94(2)$ & 90.00 & 90.00 & 90.00 & 722. & $14(35)$ \\
\hline SA $86 / 10$ & $8.571(2)$ & $12.970(2)$ & $7.215(1)$ & 90.00 & $115.92(2)$ & 90.00 & 90.00 & 90.00 & 721. & $.36(18)$ \\
\hline & $\Delta \mathrm{a}$ & $\begin{array}{c}\mathrm{t}_{1} \mathrm{o}+ \\
\mathrm{t}_{1} \mathrm{~m}\end{array}$ & $\begin{array}{c}\mathrm{t}_{1} \mathrm{o}- \\
\mathrm{t}_{1} \mathrm{~m}\end{array}$ & $\mathrm{t}_{1} \mathrm{O}$ & $\mathrm{t}_{1} \mathrm{~m}$ & $\begin{array}{l}\mathrm{t}_{2} \mathrm{O}= \\
\mathrm{t}_{2} \mathrm{~m}\end{array}$ & $\begin{array}{c}\Delta 2 \theta \mathrm{CuK} \alpha \\
(204-060)\end{array}$ & $\begin{array}{c}\Delta 2 \Theta C u K \alpha \\
\text { d(201) }\end{array}$ & $\begin{array}{l}\% \text { Or } \\
\text { (Vol.) }\end{array}$ & $\begin{array}{l}\% \text { Or } \\
\mathrm{d}(201)\end{array}$ \\
\hline Bobbejaan & granite - & file. & & & & & & & & \\
\hline $78 / 15 / 1$ & 0.00 & 0.97 & 0.85 & 0.91 & 0.46 & 0.02 & 8.760 & 21.01 & 98.9 & 94.9 \\
\hline $78 / 15 / 4$ & 0.00 & 1.00 & 0.88 & 0.94 & 0.47 & 0.00 & 8.720 & 20.99 & - & 96.7 \\
\hline $78 / 15 / 7$ & -0.02 & 0.98 & 0.84 & 0.91 & 0.46 & 0.01 & 8.745 & 20.98 & - & 97.6 \\
\hline $78 / 15 / 10$ & -0.02 & 0.97 & 0.82 & 0.89 & 0.45 & 0.02 & 8.750 & 20.96 & - & 99.5 \\
\hline Bobbejaan & granite - n & eralized. & & & & & & & & \\
\hline $06377-288$ & 0.00 & 0.96 & 1.00 & 0.98 & 0.49 & 0.02 & 8.775 & - & - & - \\
\hline $06377-300$ & -0.05 & 1.00 & 0.84 & 0.92 & 0.46 & 0.00 & 8.735 & - & 99.5 & - \\
\hline $06377-24$ & -0.08 & 0.96 & - & - & - & - & 8.770 & - & - & - \\
\hline $06377-108$ & -0.04 & 1.00 & 0.90 & 0.95 & 0.48 & 0.00 & 8.700 & 20.96 & - & 99.5 \\
\hline $06377-154$ & -0.04 & 1.00 & 0.86 & 0.93 & 0.47 & 0.00 & 8.730 & - & 98.3 & - \\
\hline $06377-20$ & -0.03 & 0.95 & 0.80 & 0.88 & 0.44 & 0.03 & 8.780 & - & 97.4 & - \\
\hline Lease gran & & & & & & & & & & \\
\hline $06377-45$ & -0.18 & 0.91 & 0.54 & 0.73 & 0.36 & 0.05 & 8.835 & - & 59.1 & - \\
\hline $06377-139$ & -0.03 & 0.94 & 0.35 & 0.65 & 0.32 & 0.03 & 8.855 & - & 88.4 & - \\
\hline $06377-143$ & -0.08 & 1.00 & 0.31 & 0.66 & 0.33 & 0.00 & 8.805 & - & 68.4 & - \\
\hline $06377-144$ & -0.03 & 0.97 & 0.33 & 0.65 & 0.33 & 0.02 & 8.790 & - & 76.7 & - \\
\hline $06377-146$ & 0.08 & 1.00 & 0.38 & 0.69 & 0.35 & 0.00 & 8.785 & - & 95.7 & - \\
\hline $06377-160$ & -0.01 & 0.89 & 0.27 & 0.58 & 0.29 & 0.06 & 8.865 & - & 89.3 & - \\
\hline Main gran & - triclinic. & & & & & & & & & \\
\hline $\mathrm{SA} 62 / 1$ & -0.03 & 0.97 & 0.99 & 0.98 & 0.49 & 0.02 & 8.795 & - & 98.8 & - \\
\hline $\mathrm{SA} 62 / 1$ & -0.03 & 0.95 & 0.91 & 0.93 & 0.47 & 0.03 & 8.795 & - & 92.7 & - \\
\hline SA $60 / 4$ & -0.04 & 0.96 & 1.00 & 0.98 & 0.49 & 0.02 & 8.765 & - & - & - \\
\hline SA $88 / 5$ & -0.03 & 0.98 & 1.00 & 0.99 & 0.50 & 0.01 & 8.775 & - & 99.5 & - \\
\hline SA $36 / 7$ & -0.01 & 0.98 & 0.97 & 0.98 & 0.49 & 0.01 & 8.770 & - & - & - \\
\hline Main gran & - monoclini & & & & & & & & & \\
\hline SA $76 / 8$ & 0.05 & 0.84 & - & 0.42 & 0.21 & 0.08 & 8.995 & 20.92 & - & 96.3 \\
\hline SA $90 / 8$ & -0.03 & 0.88 & - & 0.44 & 0.22 & 0.06 & 8.880 & 20.96 & 98.0 & 92.8 \\
\hline $\mathrm{SA} 14 / 10$ & 0.03 & 0.85 & - & 0.43 & 0.22 & 0.08 & 8.960 & 20.89 & 97.7 & 98.9 \\
\hline SA $86 / 10$ & -0.01 & 0.93 & - & 0.47 & 0.23 & 0.04 & 8.830 & 20.95 & 92.5 & 93.6 \\
\hline
\end{tabular}




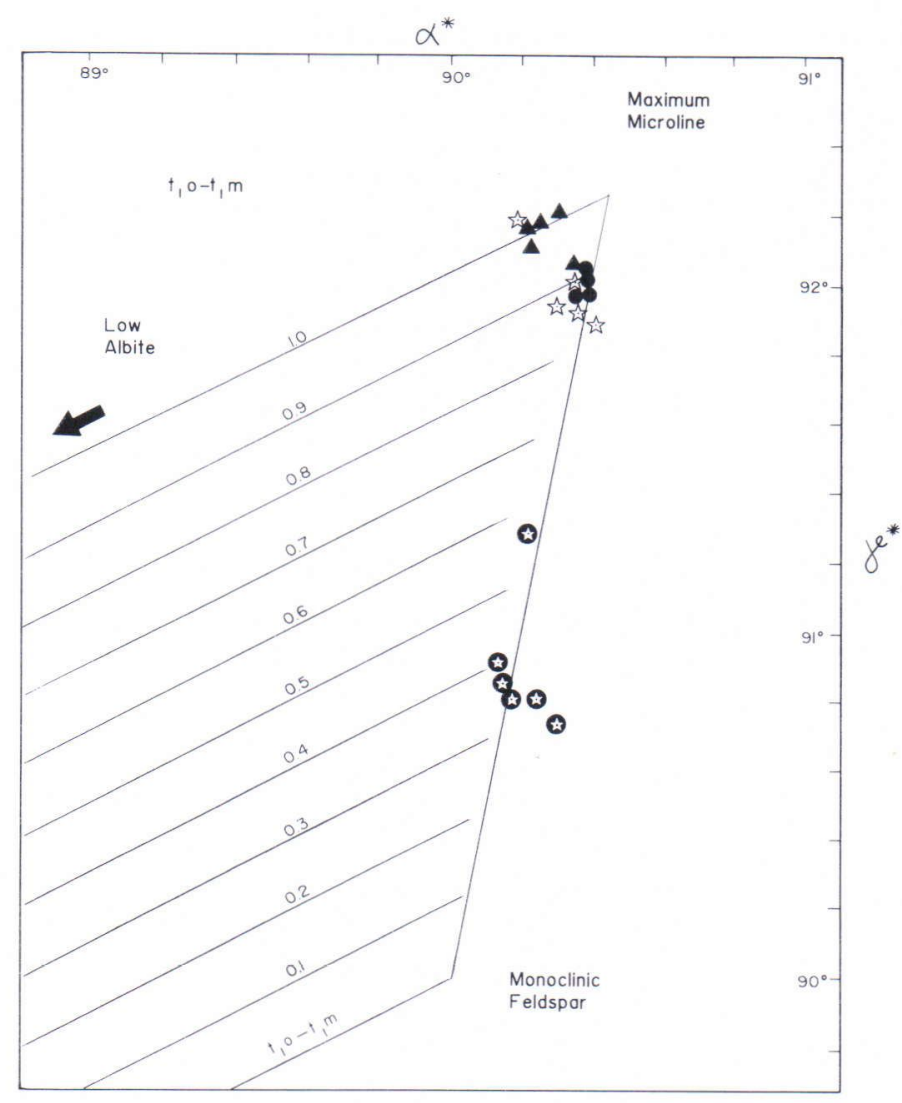

Fig. 3. $\alpha^{*}-\gamma^{*}$ plot for alkali feldspars, contoured for $\mathrm{t}_{1} \mathrm{O}-\mathrm{t}_{1} \mathrm{~m}$ with complete order in the low albite - maximum microcline series. $t_{1} \mathrm{o}$ equals $t_{1} \mathrm{~m}$ in analbite and monoclinic alkali feldspars (Stewart and Wright, 1974). Marks as in Fig. 1.

The Or-contents were estimated with a reproducibility of about two per cent from the $\overline{2} 01$ peak in the diffractometer pattern. The composition was determined by using the following equations (Wright 1968):

Orthoclase series: Or\% $=1930.77-87.69 \times$ $2 \Theta_{i 01}$

Maximum and Intermediate microcline-

Low albite series: Or\% $=2031.77-92.19 \times$ $2 \Theta_{201}$

The Or $\%$ contents determined from the $\overline{2} 01$ peak values are also given in Table 1 . The orthoclase contents cannot always be determined from the $\overline{2} 01$ peak because it overlaps with the strong peak of the quartz internal standard.

An equation of Stewart and Wright (1974) re- lating Or-content and cell volume $\left(\mathrm{V}, \AA^{3}\right)$ was applied in this study:

$$
\operatorname{Or} \%=\frac{0,2962-\sqrt{0.953131-0.0013 \mathrm{~V}}}{0.0018062}
$$

The equation is least satisfactory for higly ordered sodic feldspars (Stewart 1975) and, consequently, the Or contents calculated for most of the Bobbejaankop granite samples and for three of the Main granite were slightly over 100 per cent.

\section{Geothermometry and oxygen barometry using coexisting iron-titanium oxides}

All the specimens studied are from the coarsegrained lower portion of the Main granite, from 
between 1215 and 1650 metres above sea level in the environment of the Zaaiplaats tin mine. The textural relationship between magnetite and ilmenite is shown in Fig. 4.

Coexisting magnetite and ilmenite grains could not be found in the upper, porphyritic part of the Main granite or in the Bobbejaankop or Lease granites. In the porphyritic Main granite most of the ilmenite is usually altered to leucoxene; in the Bobbejaankop granite all the magnetite has oxidized into hematite, and ilmenite could not be identified in polished sections. In the original microprobe analyses all the iron is given as $\mathrm{FeO}$; the $\mathrm{Fe}_{2} \mathrm{O}_{3}$ contents were estimated using charge balance criteria (Carmichael 1967). The maximum and minimum temperatures, calculated with the method of Powell and Powell (1977), of each analysed magnetiteilmenite pair are given in Table 2. The temperatures display fairly random distribution patterns between $450^{\circ} \mathrm{C}$ and $750^{\circ} \mathrm{C}$, the average temperature being $600^{\circ} \mathrm{C}$. One magnetiteilmenite pair in Specimen SA 49 gave temperatures as high as $946.9^{\circ} \mathrm{C}$ and $963.6^{\circ} \mathrm{C}$, whereas one single pair in Specimen SA 96 gave the lowest temperatures calculated, $377.8^{\circ} \mathrm{C}$ and $387.8^{\circ} \mathrm{C}$.

The temperatures obtained with the graphical method of Buddington and Lindsley (1964) cor-

Fig. 4. Photomicrographs of the textural relationships of the magnetite-ilmenite pairs analysed. The length of the scale bars is $100 \mu \mathrm{m}$. A. Coexisting magnetite (Mt) and ilmenite (In) in a grey, fairly coarse-grained Main granite. Ilmenite occurs as ehedral, sub-rounded grains on the edges of larger magnetite crystals. In a few cases ilmenite grains are not in immediate contact with magnetite. Sample SA 141. B. A typical mode of occurrence of large anhedral ilmenite (In) grains with magnetite (Mt). The small blebs of hematite inclusions are the result of martitization. The smaller ilmenite grains tend to form sharp edges towards the larger magnetite crystals. Although the contact between the two minerals are irregular no indication of their formation as a result of exsolution can be confirmed. Ilmenite, however, occurs more often as inclusions in magnetite although magnetite was also found within the ilmenite grains. Both magnetite and ilmenite grains may have irregular silicate inclusions. The host granite is a reddish, coarse-grained variation of the Main granite. Sample SA 96.
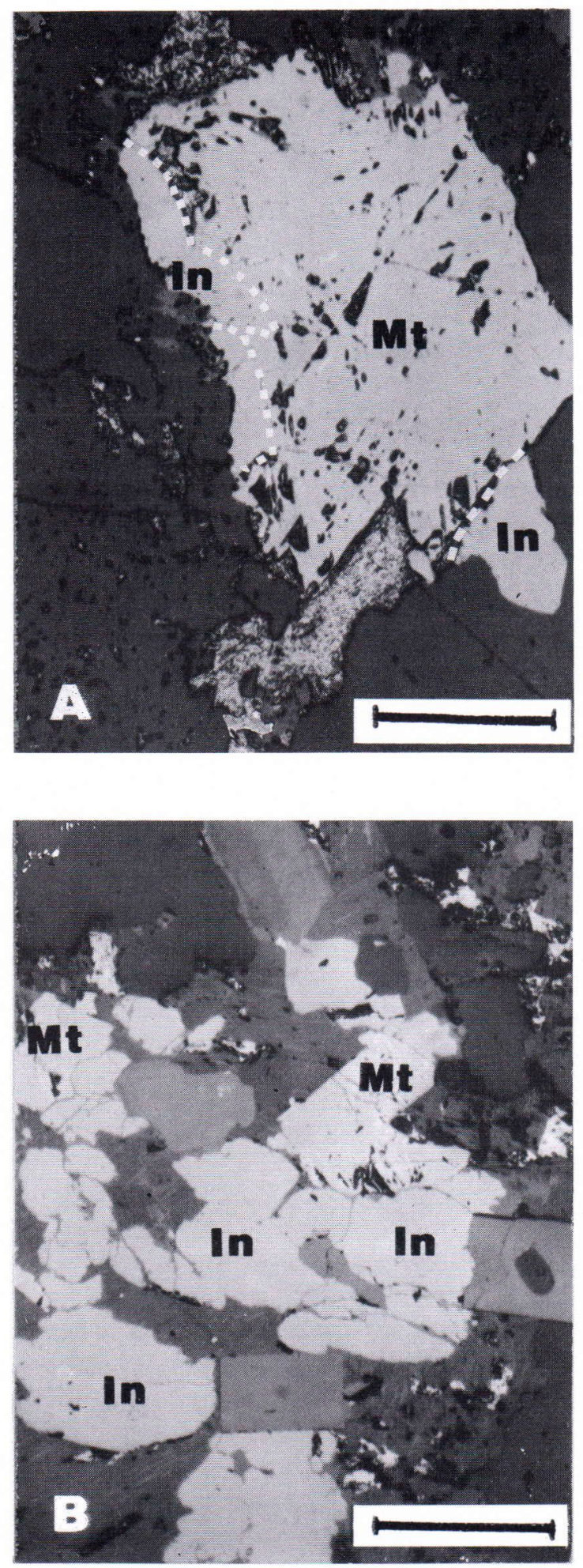
Table 2. Microprobe analyses of coexisting magnetite and ilmenite from the Main granite in the Zaaiplaats area.

\begin{tabular}{|c|c|c|c|c|c|c|c|c|c|c|c|c|c|c|c|}
\hline Specimen & SA 49 a & SA $49 \mathrm{~b}$ & SA $49 \mathrm{c}$ & SA $49 \mathrm{~d}$ & SA $49 \mathrm{e}$ & SA $49 \mathrm{f}$ & SA $49 \mathrm{~g}$ & SA $49 \mathrm{~h}$ & SA $49 \mathrm{i}$ & SA 141 a & $\mathrm{SA} 141 \mathrm{~b}$ & SA $141 \mathrm{c}$ & SA $141 \mathrm{e}$ & SA $141 \mathrm{e}$ & SA $141 \mathrm{f}$ \\
\hline \multicolumn{16}{|c|}{ MAGNETITE ANALYSES } \\
\hline $\mathrm{SiO}_{1}$ & 0.64 & 2.57 & 0.81 & - & - & - & - & 0.43 & 3.64 & 0.06 & 1.09 & 0.13 & 0.36 & 0.13 & 0.04 \\
\hline $\mathrm{TiO}_{2}$ & 2.77 & 4.44 & 3.03 & 2.29 & 2.43 & 3.01 & 2.94 & 3.26 & 16.83 & 5.72 & 6.73 & 2.14 & 0.56 & 1.22 & 2.86 \\
\hline $\mathrm{Al}_{2} \mathrm{O}_{3}$ & 0.39 & 0.71 & 0.58 & 0.42 & 0.44 & 0.58 & 0.49 & 0.55 & 1.27 & 0.37 & 0.44 & 0.40 & 0.21 & 0.18 & 0.50 \\
\hline $\mathrm{Cr}_{2} \mathrm{O}_{3}$ & - & - & - & - & - & - & - & - & - & - & - & - & - & - & - \\
\hline $\mathrm{FeO}$ & 88.49 & 84.57 & 87.88 & 89.54 & 89.00 & 89.33 & 89.93 & 88.21 & 66.81 & 86.19 & 83.11 & 88.57 & 90.05 & 89.23 & 88.72 \\
\hline $\mathrm{MnO}$ & 0.17 & 0.18 & 0.17 & 0.06 & 0.05 & 0.11 & 0.09 & 0.22 & 0.06 & 0.79 & 0.98 & 0.31 & 0.08 & 0.17 & 0.39 \\
\hline $\mathrm{MgO}$ & - & - & - & - & - & - & - & 0.01 & 0.04 & 0.02 & 0.23 & - & - & - & \\
\hline $\mathrm{CaO}$ & 0.32 & 1.70 & 0.52 & - & - & - & - & - & 0.29 & - & 0.12 & - & - & - & - \\
\hline TOTAL & 92.80 & 94.17 & 92.99 & 92.32 & 91.93 & 93.03 & 93.05 & 92.65 & 88.94 & 93.14 & 92.71 & 91.54 & 91.26 & 90.94 & 92.51 \\
\hline \multicolumn{16}{|c|}{ Recalculated analyses (see text) } \\
\hline $\mathrm{Fe}_{2} \mathrm{O}_{3}$ & 60.97 & 53.57 & 59.96 & 63.12 & 62.51 & 61.96 & 62.64 & 60.14 & 48.51 & 56.56 & 51.60 & 62.57 & 65.29 & 64.29 & 61.89 \\
\hline $\mathrm{FeO}$ & 33.63 & 36.37 & 33.93 & 32.75 & 32.75 & 33.58 & 33.36 & 34.10 & 20.33 & 35.30 & 36.67 & 32.28 & 31.31 & 31.38 & 33.04 \\
\hline TOTAL & 98.89 & 99.54 & 99.00 & 98.64 & 98.19 & 99.24 & 99.33 & 98.71 & 90.98 & 98.82 & 97.87 & 97.82 & 97.90 & 97.37 & 28.71 \\
\hline \multicolumn{16}{|c|}{ IL MENITE ANALYSES } \\
\hline $\mathrm{SiO}_{2}$ & 0.07 & - & 0.53 & 0.27 & 13.88 & - & 0.68 & 0.39 & 0.04 & - & 7.28 & 0.02 & 0.80 & 0.70 & - \\
\hline $\mathrm{TiO}_{2}$ & 45.83 & 46.65 & 44.40 & 47.00 & 33.66 & 47.57 & 44.42 & 44.38 & 46.83 & 47.51 & 44.16 & 49.19 & 42.71 & 33.82 & 37.24 \\
\hline $\mathrm{Al}_{2} \mathrm{O}_{3}$ & 0.07 & 0.04 & 0.52 & 0.24 & 4.81 & 0.10 & 0.37 & 0.16 & 0.14 & 0.08 & 0.02 & 0.07 & 0.24 & 0.38 & 0.03 \\
\hline $\mathrm{Cr}_{2} \mathrm{O}_{3}$ & - & - & - & - & - & - & - & - & - & - & - & - & - & - & - \\
\hline $\mathrm{FeO}$ & 46.92 & 48.04 & 45.49 & 48.04 & 40.82 & 48.14 & 45.09 & 45.88 & 47.68 & 46.18 & 43.78 & 45.45 & 44.12 & 56.68 & 55.05 \\
\hline $\mathrm{MnO}$ & 3.62 & 3.75 & 3.48 & 3.53 & 2.78 & 3.53 & 3.54 & 3.78 & 3.51 & 5.84 & 5.10 & 6.12 & 5.74 & 4.87 & 3.74 \\
\hline $\mathrm{MgO}$ & - & 0.01 & 0.01 & 0.01 & 0.10 & - & - & 0.02 & - & 0.02 & - & 0.04 & 0.05 & 0.08 & - \\
\hline $\mathrm{CaO}$ & 0.82 & - & 1.37 & - & 0.92 & - & 1.29 & 1.46 & - & - & - & - & 0.01 & - & - \\
\hline TOTAL & 97.34 & 98.49 & 96.08 & 99.09 & 96.96 & 99.33 & 95.37 & 96.06 & 98.19 & 99.63 & 100.34 & 98.96 & 93.65 & 96.43 & 96.06 \\
\hline \multicolumn{16}{|c|}{ Recalculated analyses } \\
\hline $\mathrm{Fe}_{2} \mathrm{O}_{3}$ & 7.63 & 7.32 & 7.09 & 6.58 & -3.90 & 6.57 & 6.90 & 8.25 & 6.65 & 6.93 & 0.38 & 6.76 & 7.78 & 22.33 & 18.77 \\
\hline $\mathrm{FeO}$ & 40.06 & 41.45 & 39.09 & 42.12 & 44.33 & 42.23 & 38.88 & 38.46 & 41.69 & 39.95 & 43.44 & 39.36 & 37.12 & 36.49 & 38.16 \\
\hline TOTAL & 98.09 & 99.22 & 96.79 & 99.75 & 96.58 & 100.00 & 96.08 & 96.90 & 98.87 & 100.33 & 100.38 & 99.64 & 94.45 & 98.67 & 97.94 \\
\hline $\mathrm{x}_{\mathrm{ulv}, \text { max }}$ & 0.0807 & 0.1268 & 0.0881 & 0.0672 & 0.0716 & 0.0877 & 0.0798 & 0.0953 & 0.5184 & 0.1669 & 0.1968 & 0.0633 & 0.0165 & 0.0724 & 0.0838 \\
\hline $\mathrm{x}_{\mathrm{mt}, \min }$ & 0.8700 & 0.6907 & 0.8451 & 0.9249 & 0.9204 & 0.9003 & 0.9102 & 0.8723 & 0.2962 & 0.7985 & 0.7330 & 0.9171 & 0.9636 & 0.9501 & 0.8945 \\
\hline $\mathrm{x}_{\mathrm{mt}, \max }$ & 0.8889 & 0.7656 & 0.8723 & 0.9268 & 0.9220 & 0.9040 & 0.9132 & 0.8800 & 0.3134 & 0.8255 & 0.7546 & 0.9275 & 0.9662 & 0.9560 & 0.9074 \\
\hline $\mathrm{x}_{\mathrm{ulv}, \min }$ & 0.0713 & 0.0894 & 0.0945 & 0.0663 & 0.0708 & 0.0858 & 0.0783 & 0.0914 & 0.5098 & -0.1534 & 0.1860 & 0.0581 & 0.0152 & 0.0696 & 0.0774 \\
\hline $\mathrm{x}_{\mathrm{ilm}, \max }$ & 0.7949 & 0.8628 & 0.7689 & 0.8289 & 0.2584 & 0.8380 & 0.7693 & 0.7532 & 0.8421 & 0.8927 & 0.7099 & 0.7753 & 0.7355 & 0.5643 & 0.6635 \\
\hline $\mathrm{x}_{\mathrm{hem}, \min }$ & 0.0743 & 0.0708 & 0.0663 & 0.0616 & 0.0012 & 0.0625 & 0.0660 & 0.0805 & 0.0637 & 0.0659 & 0.0034 & 0.0647 & 0.0774 & 0.2209 & 0.1884 \\
\hline $\mathrm{x}_{\text {hem, max }}$ & 0.0748 & 0.0710 & 0.0703 & 0.0634 & 0.0370 & 0.0632 & 0.0688 & 0.0817 & 0.0648 & 0.0665 & 0.1062 & 0.0653 & 0.0793 & 0.2239 & 0.1887 \\
\hline $\mathrm{x}_{\mathrm{ilm}, \min }$ & 0.7949 & 0.8628 & 0.7689 & 0.8289 & 0.2584 & 0.8380 & 0.7693 & 0.7532 & 0.8421 & 0.8927 & 0.7099 & 0.7753 & 0.7355 & 0.5643 & 0.6635 \\
\hline $\mathrm{T}_{\max }$ & 599.0 & 655.5 & 609.4 & 560.6 & 624.4 & 708.3 & 591.0 & 630.6 & 963.6 & 658.8 & 771.9 & 564.4 & 476.3 & 735.1 & 719.6 \\
\hline $\mathrm{T}_{\min }$ & 586.9 & 609.3 & 585.3 & 557.2 & 380.2 & 581.7 & 585.3 & 624.1 & 946.9 & 645.5 & 446.4 & 555.5 & 468.8 & 727.9 & 708.3 \\
\hline Av. $\mathrm{T},{ }^{\circ} \mathrm{C}$ & 593.0 & 632.4 & 597.4 & 558.9 & 502.3 & 645.0 & 588.2 & 627.4 & 955.3 & 652.2 & 609.2 & 560.0 & 472.6 & 731.5 & 714.0 \\
\hline \multirow{3}{*}{ In $\mathrm{a}_{\mathrm{O}_{2}} \begin{array}{l}\text { Min. } \\
\text { Ave. }\end{array}$} & -41.4 & -36.7 & -40.9 & -45.5 & -45.4 & -44.1 & 43.3 & -40.0 & -25.8 & -39.4 & -31.8 & -44.6 & -51.3 & -29.9 & -31.1 \\
\hline & -44.9 & -43.9 & -44.8 & -46.3 & -82.4 & -44.6 & 44.1 & -41.0 & -26.9 & -41.3 & -67.9 & -46.3 & -52.4 & -31.1 & -32.6 \\
\hline & -43.6 & -40.3 & -42.8 & -45.9 & -63.9 & -44.4 & -43.6 & -40.5 & -26.4 & -40.4 & -49.8 & -45.5 & -51.9 & -30.5 & -31.9 \\
\hline \multirow{2}{*}{$\begin{array}{l}\log _{10} \mathrm{f}_{\mathrm{O}_{2}} \\
\text { graphically } \\
\mathrm{T}\left({ }^{\circ} \mathrm{C}\right) \\
\text { graphically }\end{array}$} & -19.8 & -19.2 & -19.7 & -20.5 & -22.0 & -20.2 & -20.3 & -19.3 & -13.7 & -18.2 & -16.3 & -20.7 & - & -13.8 & -14.6 \\
\hline & $585^{\circ}$ & $620^{\circ}$ & $590^{\circ}$ & $565^{\circ}$ & $555^{\circ}$ & $580^{\circ}$ & $578^{\circ}$ & $597^{\circ}$ & $865^{\circ}$ & $650^{\circ}$ & $683^{\circ}$ & $560^{\circ}$ & - & $710^{\circ}$ & $690^{\circ}$ \\
\hline
\end{tabular}


Table 2. (continued).

\begin{tabular}{|c|c|c|c|c|c|c|c|c|c|c|c|c|c|c|c|}
\hline Specimen & SA $141 \mathrm{~g}$ & SA $141 \mathrm{~h}$ & SA $141 \mathrm{i}$ & SA 15 a & $\mathrm{SA} 15 \mathrm{~b}$ & SA $15 \mathrm{c}$ & SA 96 a & SA 96 b & SA $96 \mathrm{c}$ & SA $96 \mathrm{~d}$ & SA $96 \mathrm{e}$ & SA $96 \mathrm{f}$ & SA $96 \mathrm{~g}$ & SA 90 a & SA $90 \mathrm{~b}$ \\
\hline \multicolumn{16}{|c|}{ MAGNETITE ANALYSES } \\
\hline $\mathrm{SiO}_{2}$ & 0.16 & 0.06 & 0.22 & 10.93 & 5.53 & 2.99 & 0.59 & 1.81 & 0.01 & 0.32 & 0.51 & 0.27 & 1.34 & 0.27 & 1.49 \\
\hline $\mathrm{TiO}_{2}$ & 0.94 & 0.89 & 1.52 & 2.41 & 2.08 & 2.05 & - & 1.17 & 0.86 & - & 1.56 & 0.17 & 0.16 & 4.74 & 3.95 \\
\hline $\mathrm{Al}_{2} \mathrm{O}_{3}$ & 0.20 & 0.24 & 0.20 & 2.51 & 2.68 & 1.40 & 0.08 & 0.50 & 0.09 & 0.01 & 0.55 & 0.55 & 0.16 & 0.19 & 0.78 \\
\hline $\mathrm{Cr}_{2} \mathrm{O}_{3}$ & - & - & - & - & - & - & - & - & - & - & - & - & - & - & - \\
\hline $\mathrm{FeO}$ & 90.33 & 91.19 & 89.39 & 71.48 & 79.17 & 82.86 & 92.29 & 88.58 & 91.89 & 92.31 & 89.47 & 91.27 & 89.32 & 84.79 & 82.22 \\
\hline $\mathrm{MnO}$ & 0.30 & 0.13 & 0.25 & 0.11 & 0.04 & 0.02 & 0.04 & 0.01 & 0.16 & - & 0.39 & 0.05 & 0.09 & 1.04 & 0.28 \\
\hline $\mathrm{MgO}$ & - & - & - & 0.10 & - & - & - & - & - & 0.01 & - & - & - & 0.04 & - \\
\hline $\mathrm{CaO}$ & - & - & - & 2.05 & 1.93 & 1.66 & - & 1.24 & - & - & 0.10 & - & - & - & - \\
\hline TOTAL & 91.93 & 92.50 & 91.58 & 89.58 & 91.42 & 90.98 & 92.99 & 93.30 & 92.97 & 92.64 & 92.60 & 92.30 & 93.44 & 91.06 & 88.92 \\
\hline \multicolumn{16}{|c|}{ Recalculated analyses (see text) } \\
\hline $\mathrm{Fe}_{2} \mathrm{O}_{3}$ & 65.51 & 66.25 & 63.90 & 31.23 & 46.55 & 54.95 & 67.38 & 61.79 & 66.99 & 67.84 & 63.41 & 66.67 & 56.73 & 56.76 & 52.82 \\
\hline $\mathrm{FeO}$ & 31.38 & 31.58 & 31.90 & 43.38 & 37.28 & 33.41 & 31.67 & 32.98 & 31.61 & 31.27 & 32.42 & 31.28 & 38.28 & 33.72 & 34.70 \\
\hline TOTAL & 98.50 & 99.15 & 97.98 & 92.72 & 96.10 & 96.49 & 99.72 & 99.50 & 99.73 & 99.45 & 98.94 & 98.99 & 99.13 & 96.76 & 94.01 \\
\hline \multicolumn{16}{|c|}{ I LMENITE ANALYSES } \\
\hline $\mathrm{SiO}_{2}$ & - & - & - & - & - & - & - & - & - & - & - & - & - & - & - \\
\hline $\mathrm{TiO}_{2}$ & 45.78 & 46.96 & 45.23 & 46.43 & 45.90 & 46.36 & 48.43 & 44.65 & 45.62 & 44.87 & 46.81 & 46.97 & 46.21 & 47.31 & 46.57 \\
\hline $\mathrm{Al}_{2} \mathrm{O}_{3}$ & 0.02 & 0.03 & 0.05 & 0.07 & 0.11 & 0.07 & 0.06 & - & 0.01 & 0.04 & 0.06 & 0.02 & 0.08 & 0.08 & 0.05 \\
\hline $\mathrm{Cr}_{2} \mathrm{O}_{3}$ & - & - & - & - & - & - & - & - & - & - & - & - & - & - & - \\
\hline $\mathrm{FeO}$ & 45.46 & 45.53 & 46.88 & 46.00 & 46.10 & 45.84 & 46.65 & 44.99 & 47.94 & 46.74 & 46.81 & 46.81 & 47.45 & 44.09 & 45.68 \\
\hline $\mathrm{MnO}$ & 6.53 & 6.43 & 6.07 & 5.45 & 6.18 & 6.22 & 4.73 & 4.27 & 4.41 & 4.64 & 4.72 & 4.63 & 4.45 & 7.29 & 5.84 \\
\hline $\mathrm{MgO}$ & 0.02 & - & - & - & - & - & - & - & - & - & 0.01 & 0.05 & - & - & 0.02 \\
\hline $\mathrm{CaO}$ & - & - & - & - & - & - & - & - & - & - & - & - & - & - & - \\
\hline TOTAL & 97.81 & 98.94 & 98.24 & 97.94 & 98.28 & 98.52 & 99.87 & 96.91 & 97.99 & 99.29 & 98.08 & 98.47 & 98.18 & 98.97 & 98.16 \\
\hline \multicolumn{16}{|c|}{ Recalculated analyses } \\
\hline $\mathrm{Fe}_{2} \mathrm{O}_{3}$ & 8.10 & 7.26 & 9.13 & 7.20 & 8.16 & 7.73 & 5.82 & 9.01 & 8.43 & 2.72 & 7.02 & 6.92 & 7.67 & 6.58 & 7.20 \\
\hline $\mathrm{FeO}$ & 38.17 & 39.00 & 38.67 & 39.52 & 38.76 & 38.92 & 41.42 & 39.88 & 40.36 & 44.29 & 40.49 & 40.58 & 40.55 & 38.17 & 39.20 \\
\hline TOTAL & 98.62 & 99.68 & 99.15 & 98.67 & 99.11 & 99.29 & 100.45 & 97.81 & 98.33 & 99.56 & 99.11 & 99.17 & 98.96 & 99.43 & 98.88 \\
\hline $\mathrm{x}_{\mathrm{ulv}, \max }$ & 0.0276 & 0.0260 & 0.0448 & 0.0713 & 0.0611 & 0.0611 & 0.0000 & 0.0674 & 0.0249 & 0.0000 & 0.0455 & $0.0050^{\circ}$ & 0.0465 & 0.1412 & 0.1207 \\
\hline$x_{\mathrm{mt}, \min }$ & 0.9534 & 0.9640 & 0.9351 & 0.3661 & 0.6803 & 0.7489 & 0.9759 & 0.8388 & 0.9681 & 0.9875 & 0.9098 & 0.9750 & 0.7916 & 0.8104 & 0.7977 \\
\hline$x_{\operatorname{mt,max}}$ & 0.9633 & 0.9682 & 0.9437 & 0.4623 & 0.7625 & 0.8202 & 0.9773 & 0.8899 & 0.9734 & 0.9870 & 0.9268 & 0.9760 & 0.8343 & 0.8454 & 0.8072 \\
\hline $\mathrm{x}_{\mathrm{ulv}, \min }$ & 0.0227 & 0.0239 & 0.0405 & 0.0232 & 0.0200 & 0.0255 & -0.0007 & 0.0419 & 0.0222 & -0.0002 & 0.0370 & 0.0042 & 0.0252 & 0.1237 & 0.1159 \\
\hline $\mathrm{x}_{\mathrm{ilm} \text {,max }}$ & 0.7498 & 0.7666 & 0.7476 & 0.8256 & 0.7574 & 0.7633 & 0.8234 & 0.7865 & 0.7933 & 0.9628 & 0.8050 & 0.8073 & 0.8019 & 0.7555 & 0.7772 \\
\hline $\mathrm{x}_{\mathrm{hem}, \min }$ & 0.0790 & 0.0699 & 0.0884 & 0.0697 & 0.0785 & 0.0745 & 0.0973 & 0.0890 & 0.0823 & 0.0271 & 0.0677 & 0.0670 & 0.0741 & 0.0629 & 0.0698 \\
\hline$x_{\text {hem, } \max }$ & 0.0791 & 0.0701 & 0.0889 & 0.0703 & 0.0794 & 0.0750 & 0.0977 & 0.0890 & 0.0823 & 0.0274 & 0.0682 & 0.0671 & 0.0746 & 0.0636 & 0.0701 \\
\hline $\mathrm{x}_{\mathrm{ilm}, \min }$ & 0.7498 & 0.7666 & 0.7476 & 0.8256 & 0.7574 & 0.7633 & 0.8234 & 0.7865 & 0.7933 & 0.9628 & 0.8050 & 0.8073 & 0.8019 & 0.7555 & 0.7772 \\
\hline $\mathrm{T}_{\max }$ & 512.4 & 426.6 & 562.7 & 665.2 & 608.1 & 593.0 & - & 604.8 & 502.5 & - & 538.0 & 387.8 & 555.5 & 652.4 & 644.9 \\
\hline$T_{\min }$ & 497.5 & 491.4 & 552.9 & 538.3 & 687.6 & 512.1 & - & 557.1 & 493.8 & - & 579.6 & 377.8 & 505.7 & 633.3 & 639.2 \\
\hline \multirow{2}{*}{$\begin{aligned} & \text { Av. } \text { T, }{ }^{\circ} \mathrm{C} \\
& \text { Max. }\end{aligned}$} & 505.0 & 459.0 & 557.8 & 601.8 & 647.9 & 552.6 & - & 581.0 & 498.2 & - & 558.8 & 382.8 & 530.6 & 642.9 & 642.1 \\
\hline & -45.6 & -48.8 & -43.1 & -29.1 & -53.8 & -36.7 & - & -37.9 & -46.8 & - & -45.1 & -66.5 & -40.5 & -39.6 & -39.9 \\
\hline \multirow{2}{*}{$\begin{array}{ll}\text { In } \mathrm{a}_{\mathrm{O}_{2}} \text { Min. } & \text { Ave. } \\
\log _{2} & \end{array}$} & -55.0 & -49.9 & -44.7 & -50.8 & -55.4 & -52.4 & - & -45.2 & -48.8 & - & -48.9 & -68.4 & -51.3 & -42.5 & -40.8 \\
\hline & -50.3 & -49.4 & -43.9 & -39.9 & -54.6 & -44.6 & - & -41.6 & -47.8 & - & -47.0 & -67.5 & -45.9 & -41.1 & -40.4 \\
\hline \multirow{2}{*}{$\begin{array}{l}\log _{10} \mathrm{O}_{2} \\
\text { graphically } \\
\mathrm{T}\left({ }^{\circ} \mathrm{C}\right) \\
\text { graphically } \\
\end{array}$} & - & - & -20.6 & -20.0 & -19.8 & -20.1 & - & -19.6 & - & - & -21.0 & - & -20.8 & -18.7 & -18.9 \\
\hline & - & - & $555^{\circ}$ & $580^{\circ}$ & $575^{\circ}$ & $560^{\circ}$ & - & $580^{\circ}$ & - & - & $540^{\circ}$ & - & $545^{\circ}$ & $628^{\circ}$ & $625^{\circ}$ \\
\hline
\end{tabular}


respond reasonably well with the calculated ones (see Table 2). For temperatures below $600^{\circ} \mathrm{C}$ the graphical method is nevertheless inaccurate owing to the extrapolation of the intersecting contours for magnetite-ulvöspinel and hematite-ilmenite solid-solutions, for temperatures below about $540^{\circ} \mathrm{C}$ it cannot be used at all.

It should be noted that the maximum and minimum values for oxygen fugacities calculated on the basis of the equation of Powell and Powell (1977) who reformulated the data of Buddington and Lindsley, are given in Table 2 as $\ln \mathrm{a}_{\mathrm{O}_{2}}$, whereas the oxygen fugacities in the graphical method of Buddington and Lindsley (1964) are given as $\log _{10} f_{\mathrm{O}_{2}}$ in Table 2. Like with the temperatures, the graphical method cannot be applied to magnetite-ilmenite pairs giving temperatures below about $540^{\circ} \mathrm{C}$.

The $\log _{10} \mathrm{f}_{\mathrm{O}_{2}}$ values obtained with the graphical method are between -22.0 and -13.7 , and the calculated $\ln \mathrm{a}_{\mathrm{O}_{2}}$ values between -82.4 and -25.8 .

In Fig. 5 the temperatures calculated with the method of Powell and Powell (1977) have been plotted against the $\log _{10} \mathrm{f}_{\mathrm{O}_{2}}$ scale; the QFM buffer curve for the oxidation reaction $3 \mathrm{Fe}_{2} \mathrm{SiO}_{4}+$ $\mathrm{O}_{2}=2 \mathrm{Fe}_{3} \mathrm{O}_{4}+2 \mathrm{SiO}_{2}$ has been plotted for comparison. The calculated temperatures indicate that the subsolidus reequilibration of irontitanium oxides ceased at a very low temperature $\left(382.8^{\circ} \mathrm{C}\right)$. With the exception of four irontitanium oxide pairs, i.e. SA 49f, SA 141b, SA $15 \mathrm{~b}$ and SA $49 \mathrm{e}$, the distribution of the temperature- $\log _{10} \mathrm{f}_{\mathrm{O}_{2}}$ values between the different specimens displays a typical $\mathrm{T}-\log _{10} \mathrm{f}_{\mathrm{O}_{2}}$ plot of a rock from an acid intrusive suite (Haggerty 1976).

The lower Main granite crystailized under variable oxygen fugacities in the temperature range $500^{\circ}$ to $650^{\circ} \mathrm{C}$ and at least partially, under reducing conditions (Fig. 5). Oxidizing conditions prevailed at temperatures higher than $650^{\circ} \mathrm{C}$. Because of the slow cooling, the fairly wide temperature variations obtained for one individual specimen are usual for the $\mathrm{T}-\log _{10} \mathrm{f}_{\mathrm{O}_{2}}$ distributions for coexisting magnetite-ilmenite pairs in the acid intrusive rock suites. Thus, the temperature and oxygen fugacity values calculated apparently reflect the conditions under which the iron-titanium oxide pairs analysed were originally frozen.

\section{Discussion and conclusions}

The origin and the mode of crystallization of the Bobbejaankop and Main granites have been the most controversial point in the different models attempting to explain the evolution of the acid phase of the Bushveld Complex in the Zaaiplaats area. All the Bushveld granites in this area are extremely homogeneous in their major element geochemistry.

De Waal (1972) proposed that the Main granite should be replaced by »Main Suite», because his field data indicated that the Main granite is composed of a series of rock types, ranging from quartz porphyry, through granophyre to a coarse-grained granitic rock.

The Main granite rocks of the Zaaiplaats area in de Waal's model represent original epicrustal quartz and granite porphyry sheets that intruded along the contact of the Transvaal sediments and overlying felsite before to the emplacement of the layered mafic sequence of the Bushveld Complex. On intrusion of the mafic magma, the porphyry became metamorphosed to an increasing degree giving rise to the pseudo-stratification of the granophyres and granites. The Bobbejaankop granite, on the other hand, is a crystallization product of a magma of unknown origin and the Lease granite a highly metamorphosed and metasomatised granophyre of the Main granite.

According to Lenthall and Hunter (1977) and McCarthy and Hasty (1976) the Bushveld granites in the Potgietersrus area formed by in situ fractional crystallization of a single parent magma of crustal origin (Davies et al. 1970) and the graphic textures characteristic of the upper part 
Fig. 5. T- $\log _{10} \mathrm{f}_{\mathrm{O}_{2}}$ plot for the Main granite specimens as compared with the QFM buffer for the oxidation reaction $3 \mathrm{Fe}_{2} \mathrm{SiO}_{4}+\mathrm{O}_{2}=$ $2 \mathrm{Fe}_{3} \mathrm{O}_{4}+3 \mathrm{SiO}_{2}$. The error bars connect the maximum and minimum temperature and oxygen fugacity values calculated with the method of Powell and Powell (1977).

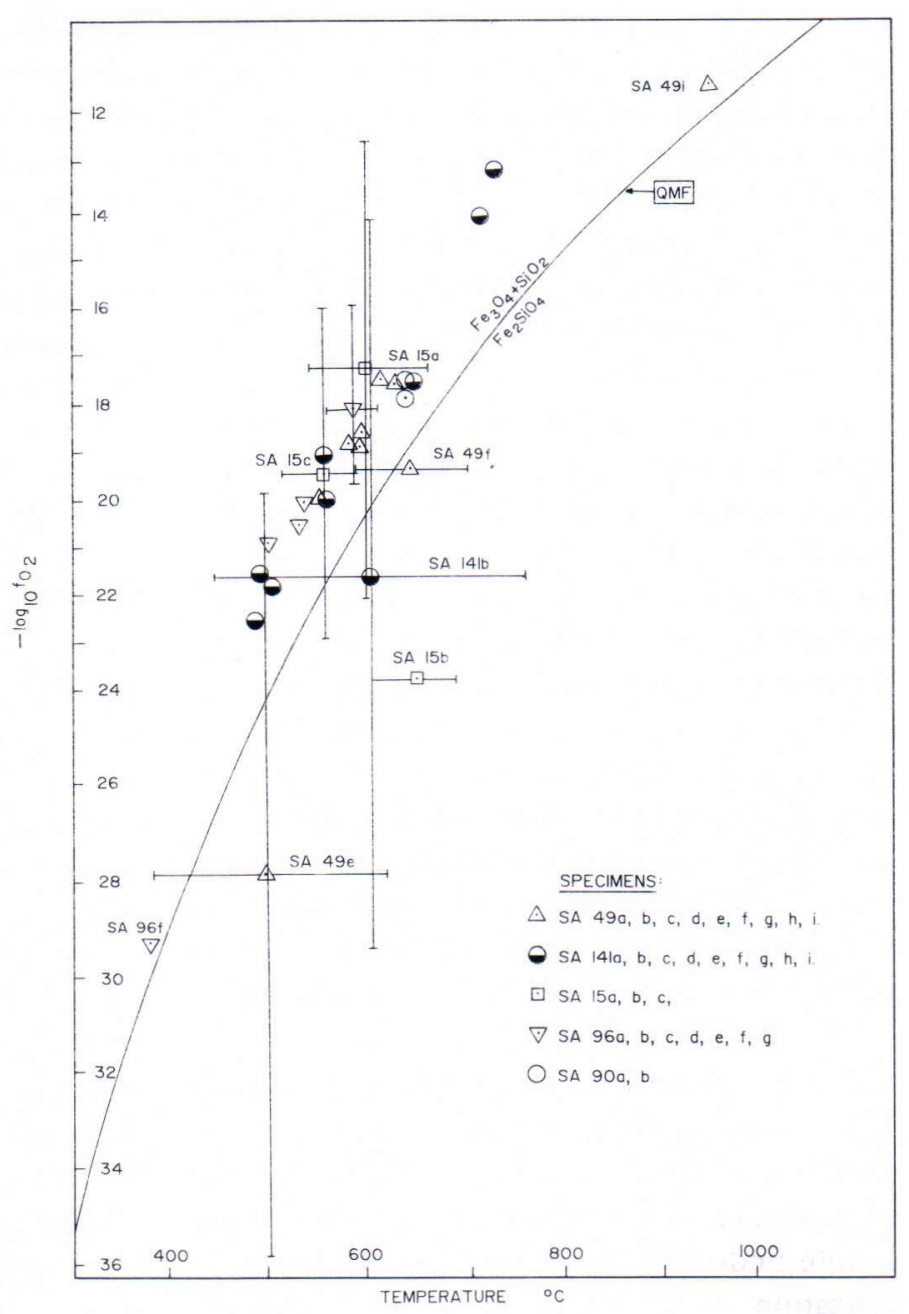

of the Main granite could result from crystallization under hydrothermal conditions or from metasomatism. In their model, the Bobbejaankop granite is regarded, on the basis of trace element partitioning at fractional crystallization, as a highly differentiated liquid caught between two fronts of crystallization: one from the roof downwards and the other from the floor upwards in a sheet of Main granite. The Lease granite is regarded as a chilled residual liquid.

The absence of oxidation and alteration phenomena in the deeper parts of the Main Granite layers may indicate crystallization from a less hydrous magma (Lenthall 1975). Further, Lenthall and Hunter (1977) argued that the alkali feldspars in the Main granite tend to achieve higher degree of ordering at stratigraphically higher levels. The present study, however, showed that the alkali feldspars in all the porphyry and porphyritic granophyre variations of the Main granite, with granularity and texture index of de Waal (1972) either seven or lower, are maximum microclines.

The high thermal states of alkali feldspars in the lower Main granite indicate the presence of only small quantities or total absence of a vola- 
tile phase at the time of initial crystal growth, which might have prevented the later development of higher ordering. This supports the view that this part of the Main granite underwent fractional crystallization. The iron-titanium oxides in the lower Main granite are original magmatic phases, and indicate a slow cooling rate under conditions typical of a rock of an acid intrusive suite.

The variation in the structural state of the alkali feldspars is mainly due to the availability of hydrothermal fluids during their cooling history (Parsons 1978). This diversity, in turn, reflects a buildup of water with magmatic evolution and implies that the fluids did not circulate freely in the lower Main Granite in the temperature range of inmixing and ordering because feldspar-fluid interactions in rocks normally tend to increase the $\mathrm{Al} / \mathrm{Si}$ ordering. However, for lack of petrographic evidence of a cumulate character it is difficult to deduce whether the crystallization was fractional or incremental equilibrium crystallization in character (McCarthy and Fripp 1980).

The high ordering of alkali feldspars, the alteration of the ferromagnesian minerals and the oxidation of exsolved iron in the feldspars could be explained if the upper layers in the Main granite underwent essentially equilibrium crystallization in the presence of a volatile-rich, hydrous fluid phase. Thus, the alkali feldspars in upper Main granite reflect the build-up of a progressively more hydrous interstitial melt containing most of the volatiles. The interaction of this interstitial melt and early solidification products has led to thorough subsolidus recrystallization of the feldspars.

A fluid inclusion study (Ollila 1981) showed that the maximum ordering or the low thermal stage of the alkali feldspars in the Bobbejaankop granite is due to the presence of the hydrosaline fluid phase which promotes the re-equilibration of alkali feldspars, and possibly, to a lesser extent, to the slow post-crystallization cooling rate, which may also be an important factor in the nucleation of triclinic potassium feldspars. On the basis of its conversion to orthoclase in a contact metamorphic aureole, Wright (1967) has estimated $375^{\circ} \mathrm{C} \pm 50^{\circ} \mathrm{C}$ as the upper temperature limit of maximum microcline.

Although the contacts between the Lease granite and the Bobbejaankop granite are usually sharp, root-like projections of the Lease granite extend down into the other. The Bobbejaankop granite also has a somewhat higher quartz content and a slightly coarser grain size, sometimes with sparsely developed pegmatitic material in the vicinity of the contact between the two granites (de Waal 1972).

The Lease granite defines trends of decreasing $\mathrm{Ba}$ and $\mathrm{Sr}$ with increasing $\mathrm{Rb}$, and low $\mathrm{K} / \mathrm{Rb}, \mathrm{Ba} / \mathrm{Sr}$ and $\mathrm{Ba} / \mathrm{Rb}$ ratios, and it has been suggested that the relationship of the Lease granite to the Main and Bobbejaankop granites is one of melt to cumulate (McCarthy and Hasty 1976).

The Lease granite could have formed in two ways: it is either an aplite or it is of metasomatic origin, i.e. it is a replacement product of the granophyre of the Main granite or the semiconsolidated Bobbejaankop granite through the process of hydrothermal granitization.

The consolidation of the Bobbejaankop granite might have led to contraction of the granite mass and development of a narrow zone of open space at its upper contact. In these circumstances, aqueous fluid might be expelled from the crystallizing magma, carrying with it granite-forming components, essentially those able to be redeposited as an aplitic granite. Since this accumulation of the »new» magma is fairly slow, it may have taken place simultaneously with the formation of the open space.

The sharp contact between the Bobbejaankop and the Lease granite supports the view that, theoretically, a heave of separate, aplitic and very hydrous magma could have appeared during the late period of the crystallization of the Bobbejaankop granite. Even though the major element composition of the Bobbejaankop and Lease granites differs but little, the faster cooling 
rate or quenching of the Lease aplitic magma may explain certain physical differences noted by de Waal (1972) between the two granites at the time of the epigenetic tin mineralization.

An alternative and more probable explanation for the origin of the Lease granite is its development as a result of hydrothermal granitization. At the final stages of the Bobbejaankop granite crystallization, continued progressive crystallization caused enrichment of volatiles and some incompatible elements in the subsequent, aqueous fluid with increasing $\mathrm{CO}_{2}$ content. This fluid accumulated gradually below the contact pegmatite owing to its low density or to the existing pressure gradients in the semi-solid Bobbejaankop granite. The initial $\mathrm{H}_{2} \mathrm{O}$ content of the Bobbejaankop granite is important because it controls the pressure and temperature regime at which an aqueous fluid phase is evolved by »second boiling» and this in turn determines the extent of wall-rock fracturing and subsequent hydrothermal activity.

Originally, the formation of the Lease granite through metasomatism was operated by a process of coexisting crystals - aqueous fluid system. The Lease granite zone undoubtedly reached $\mathrm{H}_{2} \mathrm{O}$ saturation fairly early in its metasomatism. Partial dissolution due to crystal-aqueous fluid disequilibrium led to disruption of the solids formed earlier and finally to the aplitic, fairly fine-grained texture.

According to de Waal (1972) the Lease granite represents highly metamorphosed and metasomatized granophyre of the Main granite. However, two facts appear to contradict this hypothesis, viz: (i) the location of the contact pegmatite layers that apparently mark the upper boundary of the Bobbejaankop granite, (if the contact pegmatite crystallized between the metasomatized and unaltered Main granite, it could be expected to show more irregular patterns in shape and size), and (ii) the spatial relationship between the Bobbejaankop and Lease granites. The Lease granite occurs in the Bobbejaankop granite as inclusions and as root-like apophyses up to 120 metres long (Strauss 1954). The occur- rence of the metasomatised Main granite about 120 metres beneath the roof contact is rather unlikely.

The incipient pegmatization seen on microscopic scale in the otherwise hypidiomorphic Lease granite shows a typical replacement feature formed via the later aqueous fluid phase. Although, texturally, parts of the Lease granite appear locally to be a replacement product caused by the aqueous fluid phase, or a remnant of palingenesis, of the Main granite granophyre, it is most probably metasomatized Bobbejaankop granite. If the upward crystallization from the bottom of the Bobbejaankop granite dominated over the downward crystallization from the roof, the remaining silicate melt was inevitably enriched in $\mathrm{H}_{2} \mathrm{O}$. The root-like apophyses of the Lease granite down to the Bobbejaankop granite may be the channelways for the collection of the aqueous fluid up to the Lease granite zone by convective-type circulation. Because of the loss of heat to the solid or semisolid country rock, the crystals - aqueous fluid system in the Lease granite zone was no longer thermodynamically stable at certain temperatures, and reconstitution of the Bobbejaankop granite resulted.

The primary and secondary fluid inclusions (Ollila 1981) indicated that a highly saline fluid phase was present during the cooling of the Lease granite in much the same way as in the Bobbejaankop granite. Thus, the higher thermal or less ordered state of the alkali feldspars is solely due to the higher cooling rate of the Lease granite than that of the surrounding rocks, as a consequence of sudden loss of heat from the fluid phase into the country rocks.

Acknowledgements. This investigation is part of a Ph.D. project supervised by Prof. Johan de Villiers, and accomplished with the Applied Mineralogy Research Group of MINTEK (Council for Mineral Technology, Randburg, South Africa). The author wishes to thank Prof. de Villiers and Dr. Martti Lehtinen of the University of Helsinki for their helpful criticism of the manuscript, and the president of MINTEK for permission to publish this paper. 


\section{References}

Appleman, D. E. \& H. T. Evans, Jr., 1973. Job 9214: Indexing and least-squares refinement of poder diffraction data. Natl. Tech. Inf. Serv., U.S. Dep. Commerce, Springfield, Virginia, Document PB2-16188, 67 p.

Buddington, A. F. \& D. H. Lindsley, 1964. Iron-titanium oxide minerals and synthetic equivalents. J. Petrol 5 , 310-357.

Carmichael, I. S. E., 1967. The iron-titanium oxides of salic volcanic rocks and their associated ferromagensian silicates. Contr. Miner. Petrol. 14, 36-64.

Davies, R. D., H. L. Allsopp, A. J. Erlank \& W. I. Mouton, 1970. $\mathrm{Sr}$ isotope studies on various layered intrusions in Southern Africa. Geol. Soc. S. Afr. Spec. Publ. 1, Editors: D. Visser and G. von Gruenewaldt, 579-593.

De Waal, S. A., 1972. The Bushveld granites in the Zaaiplaats area. Geol. Soc. S. Afr. Trans. 76, 135-148.

Haggerty, S. E., 1976. Opaque mineral oxides in terrestrial igneous rocks. In: Miner. Soc. Am. Short Course Notes, Oxide Minerals. Editor: Rumble, D. III, Hg-101-300.

Lenthall, D. H., 1975. Aspects of the geochemistry of the acid phase of the central and eastern Bushveld Complex. Econ. Geol. Res. Unit, Univ. Witwatersrand, Johannesb., Info. Circ. 99, 20 p.

Lenthall, D. H. \& D. R. Hunter, 1977. The geochemistry of the Bushveld granites in the Potgietersrus tin-field. Precambrian Res. 5, 359-400.

McCarthy, T. S. \& R. E. P. Fripp, 1980. The crystallization history of a granitic magma, as revealed by trace element abundances. J. Geol. 88, 211-224.

McCarthy, T. S. \& R. A. Hasty, 1976. Trace element distribution patterns and their relationship to the crystallization of granitic melts. Geochim. Cosmochim. Acta 40, $1351-1358$.

Ollila, J. T., 1981. A fluid inclusion and mineralogical study of tin deposits and rocks associated with the Bushveld Complex at the Zaaiplaats, Rooiberg and Union tin mines in the Central Transvaal, South Africa. Ph.D. thesis, Rand Afrikaans Univ., Johannesb., 257 p.

Parsons, I., 1978. Feldspars and fluids in cooling plutons. Miner. Mag. 42, 1-17.

Powell, R. \& M. Powell, 1977. Geothermometry and oxygen barometry using coexisting iron-titanium oxides: a reappraisal. Miner. Mag. 41, 257-263.

Stewart, D. B., 1975. Lattice parameters, composition, and $\mathrm{Al} / \mathrm{Si}$ order in alkali feldspars. In: Miner. Soc. Am. Short Course Notes, Feldspar Mineralogy. Editor: Ribbe, P.H., St $-1-22$.

Stewart, D. B. \& P. H. Ribbe. 1969. Structural explanation for variations in cell parameters of alkali feldspar with $\mathrm{Al} / \mathrm{Si}$ ordering. Am. J. Sci. 167-A, 444-462.

Stewart, D. B. \& T. L. Wright, 1974. Al/Si order and symmetry of natural alkali feldspars, and the relationship of strained cell parameters to bulk composition. Bull. Soc. Franc. Miner. Crist. 97, 356-377.

Strauss, C. A., 1954. The geology and mineral deposits of the Potgietersrus tin-fields. Mem. Geol. Surv. S. Afr. 46, $241 \mathrm{p}$.

Wright, T. L., 1967. The microcline - orthoclase transformation in the contact aureole of the Eldora stock, Colorado. Am. Mineral. 52, 117-136.

Wright, T. L., 1968. X-ray and optical study of alkali feldspar: II. An X-ray method for determining the composition and structural state from measurement of $2 \theta$ values for three reflections. Am. Mineral. 53, 88-104.

Wright, T. L. \& D. B. Stewart, 1968. X-ray and optical study of alkali feldspar: I. Determination of composition and structural state from refined unit-cell parameters and 2V. Am. Mineral. 53, 38-87.

Manuscript received, July 12, 1983. 